\title{
Regulatory RNAs in archaea: first target identification in Methanoarchaea
}

\author{
Daniela Prasse*, Claudia Ehlers", Rolf Backofen† and Ruth A. Schmitz"1 \\ "Institut für Allgemeine Mikrobiologie, Christian-Albrechts-Universität zu Kiel, Am Botanischen Garten 1-9, 24118 Kiel, Germany, and †Institut für Informatik, \\ Albert-Ludwigs-Universität zu Freiburg, Georges-Koehler-Allee, 79110 Freiburg, Germany
}

\begin{abstract}
sRNAs (small non-coding RNAs) representing important players in many cellular and regulatory processes have been identified in all three domains of life. In Eukarya and Bacteria, functions have been assigned for many SRNAs, whereas the SRNA populations in Archaea are considerably less well characterized. Recent analyses on a genome-wide scale particularly using high-throughput sequencing techniques demonstrated the presence of high numbers of sRNA candidates in several archaea. However, elucidation of the molecular mechanism of sRNA action, as well as understanding their physiological roles, is in general still challenging, particularly in Archaea, since efficient genetic tools are missing. The identification of cellular targets of identified archaeal sRNAs by experimental approaches or computational prediction programs has begun only recently. At present, targets have been identified for one archaeal sRNA, SRNA ${ }_{162}$ in Methanosarcina mazei, which interacts with the $5^{\prime}$ region of its targets, a cis-encoded and a trans-encoded target, blurring the paradigm of a border between cis- and trans-encoded SRNAs. Besides, the first experimental implications have been obtained in Haloarchaea and Pyrobaculum that archaeal sRNAs also target $3^{\prime}$ regions of mRNAs. The present review summarizes our current knowledge on archaeal sRNAs and their biological functions and targets.
\end{abstract}

\section{Small regulatory RNAs}

The recent discovery of an increasing number of large and small non-protein-coding RNAs with specific regulatory functions found in all three domains of life has significantly changed the view of gene expression and gene regulation (reviewed in [1-6]. In the eukaryotic domain, small RNAs (20-27 nt) belonging to two classes (microRNAs and short interfering RNAs) are known to play essential roles in development, cellular activities and physiology (for reviews, see $[5,7,8])$. In recent years, it has been shown that in bacteria sRNAs (small non-coding RNAs), which range in length from approximately 50 to $500 \mathrm{nt}$ are involved in a variety of adaptive cellular responses to biotic and abiotic stresses, and development [1,3,6,9-11]. Most of the bacterial sRNAs known today modulate translation by binding their target mRNA via imperfect sequence complementarity, resulting in either blocking the ribosomal-binding site or melting of inhibitory secondary structures that sequester the ribosomal entry site of the mRNA or effect stability of their target mRNA (for reviews, see $[1,3,11]$. Thus they appear to be frequently involved in fine-tuning of cellular responses to changing environments, indicating their important role integrated in regulons. Trans-encoded sRNAs are only partially complementary to their target (Figure 1), and the RNA-target interactions are often facilitated by the bacterial RNA chaperon Hfq [12]. Cis-encoded as (antisense) RNAs,

Key words: archaea, Methanosarcina mazei, post-transcriptional regulation, small non-coding RNA (sRNA), target gene.

Abbreviations used: as, antisense; nc, non-coding; SRNA, small non-coding RNA; TSS,

transcriptional start site; UTR, untranslated region.

${ }^{1}$ To whom correspondence should be addressed (email rschmitz@ifam.uni-kiel.de). which are encoded on the opposite strand of their target genes, are in general less well characterized compared with transencoded sRNAs. They overlap mainly with either the $5^{\prime}$ or the $3^{\prime}$ UTR (untranslated region) of their target and interact in an Hfq-independent way, which is possibly due to the long stretch of base pairing with their target mRNA (Figure 1).

\section{Identification and analysis of SRNAs in archaea}

Compared with bacteria, the archaeal sRNA population is less well characterized today, since only a few attempts have been made until recently to identify and study the role of non-coding RNAs in Archaea, the third domain of life. First, archaeal non-coding RNAs were identified in Archaeoglobus fulgidus, Sulfolobus solfataricus, Methanocaldococcus janaschii and Pyrococcus furiosus approximately 10 years ago using computational and experimental approaches. The majority of these belong to the class of C/D-box or H/ACA-box small RNAs, which participate in ribosomal RNA biogenesis and tRNA maturation [13-18]. Besides those RNAs, further novel archaeal non-coding RNAs have been identified in these early studies which are probably involved in posttranscriptional control of gene expression, including RNAs from intergenic regions and RNAs complementary to $3^{\prime}$ or $5^{\prime}$ UTRs of mRNAs or overlapping annotated open reading frames [18-21]. Additional non-coding RNAs transcribed from 12 intergenic regions have been recently identified in Pyrococcus abyssi by a combination of computational and experimental approaches, which are conserved throughout 
Figure 1 | Cis- and trans-encoded sRNAs

(A) Cis-encoded RNAs are represented by complete complementarity of the RNA duplex formed with the target mRNA (mainly 5' or 3' region). (B) Trons-encoded sRNAs form only partial complementarity of the RNA duplex with the target mRNA.

A

\section{Cis-encoded sRNA}
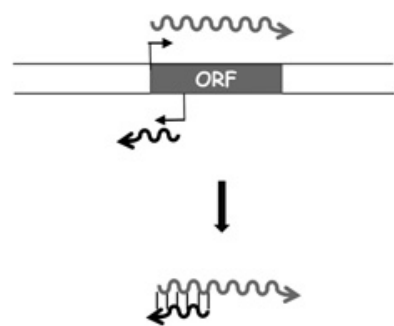

Complete complementarity

\section{Trans-encoded sRNA}
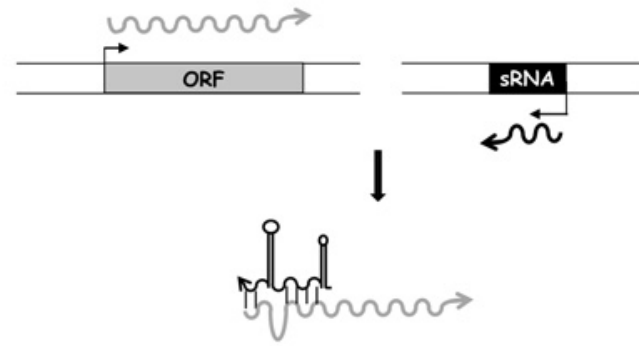

Partial complementarity the Thermococcales [22]. Analyses of sRNA populations on a genome-wide scale by RNomics, co-immunoprecipitation approaches or high-throughput sequencing techniques, e.g. the RNA-Seq approach, which has revolutionized sRNA discovery in many organisms [23-26], have demonstrated the presence of high numbers of sRNA candidates in several archaea: Methanosarcina mazei [27], S. solfataricus [20,28,29], Haloferax volcanii [30-32] and Pyrobaculum sp. [33]. Besides, studying RNA processing in the minimal archaeon Nanoarchaeum equitans by an RNA-Seq approach has recently provided an overview of the population of sRNAs in $N$. equitans [34]. Thus, to date, regulatory RNAs have been studied and reported to various degrees of complexity in only nine archaea (for further details, see [35])

\section{Post-transcriptional regulation in archaea by cis-elements}

Besides elucidating regulation on the transcriptional level, to date, generally only very little is known concerning post-transcriptional regulation in Archaea. The presence and potential regulatory function of UTRs of mRNAs (5' UTR or 3' UTR) has never been systematically studied for archaea until recently, when the genome-wide high-throughput sequencing techniques were established (see above). Beforehand, there were only three reports on experimental implications for regulatory $5^{\prime}$ UTRs of mRNAs in Archaea, which have not been further studied or confirmed: the $5^{\prime}$ leader of the mRNA encoding the DEAD-box RNA helicase of Methanococcus burtonii [36], and the methanol:CoM methyltransferase isoenzymes of Methanosarcina acetivorans ( $m$ taA1 and $m$ taA2) [37], as well as the $5^{\prime}$ leader sequence of the carbon monoxide dehydrogenase/acetyl-CoA synthase mRNA in $M$. acetivorans [38]. On the other hand, comparative analysis of genome-wide transcriptome and proteome datasets often indicated that post-transcriptional regulation is relevant in several archaea, e.g. Halobac- terium salinarum and $H$. volcanii [39] or Metbanococcus maripaludis [40]. Recent transcriptome studies via highthroughput sequencing techniques, particularly when using the differential RNA-seq method, identified high numbers of TSSs (transcriptional start sites) genome-wide, consequently allowing the characterization of the length of the respective 5 ' leaders. For example, the TSSs of 876 transcripts were identified in the genome of $M$. mazei strain Gö1 [27]. The majority of those transcripts carry unexpectedly long $5^{\prime}$ UTRs, which are up to $500 \mathrm{nt}$ in length with an average size of 150-200 nt. This finding is in contrast with other archaea such as the Haloarchaea, where most mRNAs are leaderless or contain very short leaders [41], as well as $S$. solfataricus, where the majority of mRNAs lack 5' UTRs completely [28]. The fact that $M$. mazei features long $5^{\prime}$ UTRs strongly argues for high relevance of post-transcriptional regulation at $5^{\prime}$ UTRs in methanogenic archaea, e.g. by binding regulatory proteins, RNAs (sRNAs or other mRNAs) or small metabolites, or at the level of transcript stability or translation rate. Binding metabolites and subsequent structural changes of the $5^{\prime}$ UTR upon binding would indicate the presence of a socalled riboswitch. However, except for those experimental implications in $M$. mazei, to date, no riboswitch has been identified in archaea and neither was experimental evidence obtained showing gene regulation in archaea by riboswitches. The only exception is the highly conserved riboswitch candidate $\mathrm{crcB}$ present in $M$. acetivorans upstream of the $\operatorname{crc} B$ gene, which was predicted in silico by a comparative genomics approach and potentially binds a yet unknown metabolite [42]. Furthermore, the recent study of nc (non-coding) RNAs in $P$. abyssi using computational and experimental approaches discovered the presence of four conserved ncRNAs, which appear to originate from mRNA leaders by maturation of longer transcripts [22]. Two of those ncRNAs (sRk28 and $s R k B$ ) share several features with bacterial riboswitches and are proposed to represent a novel archaeal family of ncRNAs generated by RNA processing or transcription attenuation. Besides the potential regulatory role of archaeal $5^{\prime}$ UTRs, 
some experimental findings argue for the importance of $3^{\prime}$ UTRs for regulation in Archaea, as demonstrated for two $H$. volcanii genes, where the $3^{\prime}$ UTRs have been shown to be required for translational regulation by interacting with the 5' UTR [43]. Further experimental implications have been obtained for a conserved regulatory element located in the $3^{\prime}$ UTR of the tpi gene (encoding triose phosphate isomerase) in several Pyrobaculum species (the so-called 'tpi element', see below) [33].

\section{Elucidating potential biological functions of archaeal SRNAs}

Despite the increasing number of identified sRNAs in several archaea, understanding the physiological roles of sRNAs is in general still a challenge. One powerful genetic approach to elucidate the biological function is to construct mutants of the respective sRNAs in order to evaluate the function of the sRNA under various stress conditions in comparison with the wild-type. However, although significant progress has been made in recent years, the lack of established efficient genetic tools for archaea and efficient cultivation techniques is still a bottleneck for functional analysis by genetic approaches. Thus functional analysis of regulatory components allowing detailed insights into global regulatory networks of Archaea has been performed only since genetic tools have been developed for quite a number of archaeal model systems [44]. The lack of efficient genetic tools might also be the main reason only very few archaeal sRNAs have been studied and characterized concerning their regulatory function and their targets. Those examples known today are summarized in the following.

For Halobacterium halobium, several chromosomal deletion mutants of verified intergenic sRNA-genes have been generated. The majority of those deletion mutants showed growth defects only under a few of the growth conditions tested in comparison with the parental background, e.g. under high temperatures, low salt concentrations or when growing on different carbon sources (for details, see [3032]). This strongly argues for essential roles of sRNAs in $H$. balobium in metabolic regulation; further detailed analysis including target analysis is currently underway. In M. mazei, a high number of verified sRNAs showed different expression levels in response to nitrogen availability, which might be due to direct or indirect effects [27], strongly arguing for a prominent regulatory role in nitrogen metabolism. For one of those sRNAs, designated sRNA ${ }_{154}$, direct transcriptional control by the global nitrogen transcriptional regulator NrpR has been confirmed [27]. Moreover, characterizing the respective chromosomal deletion mutant demonstrated a severe growth phenotype under nitrogen-limiting (nitrogenfixing) conditions, strongly indicating that $s \mathrm{RNA}_{154}$ plays a crucial role in the post-transcriptional regulation of nitrogen metabolism particularly under nitrogen-fixation conditions [45]. Detailed target analyses of sRNA 154 as well as elucidating the molecular mechanism of regulation are currently underway.

\section{Target analyses of sRNAs}

Despite the increasing number of sRNAs identified and understanding the physiological roles of sRNAs, the number of experimentally verified targets of sRNAs is in general considerably lower. This is due to the fact that it is still challenging to analyse the corresponding cellular targets of an identified sRNA of unknown function. Thus, although the expression of sRNAs has been verified in several archaea, neither a potential target RNA nor a molecular mechanism of target regulation has been elucidated until very recently (see below). Currently, several experimental strategies are available that have been used to identify and validate bacterial sRNA targets (mRNA targets or protein targets) (reviewed in $[26,46,47])$, including transcriptome analysis of sRNA deletion mutants or the pulse-expression of sRNAs followed by global transcriptome analysis (using microarrays), copurification of tagged sRNAs (aptamers or polyA) and novel GFP (green fluorescent protein)-reporter gene fusions $[48,49]$. The recently developed method for high-throughput sequencing and the decrease in price now allows us to analyse the targets by sequencing respective cDNA libraries of sRNA mutants or after pulse-expression of sRNAs.

In addition, several novel computational prediction programs for sRNA targets in prokaryotes have been developed in recent years using new biocomputational prediction algorithms. All target prediction tools determine regions of mutual complementarity, but differ in the way that they evaluate internal structure that might block the interaction site. Two major classes can be distinguished. The first class, with programs such as RNAup, RNAplex, IntaRNA and sTarPicker [50-54], combines the effect of intra-molecular structure into a single value called accessibility. The accessibility is the energy required to open the interaction. From the aforementioned approaches, only RNAup and IntaRNA use accessibility in its full form, with IntaRNA being fast enough for genome-wide scans. Despite their success, the above tools can predict only one continuous interaction site, excluding, e.g., double-kissing hairpins. For that reason, the second major class of prediction tools, such as biRNA [55] and RIP [56], predicts a common secondary structure for sRNA and mRNA directly, at the expense of a much higher runtime. Another possibility that was investigated for improving prediction quality is to use comparative information. On the one hand, it has been shown [57] that conservation of interaction sites is not a general feature of known targets. On the other hand, if an interaction is conserved in a multiple alignment of both sRNA and mRNA, then this is a strong signal indicating likely candidates for true interactions [58]. Interestingly, although the target should be clear, target interactions of cis-encoded asRNAs in archaea have not been experimentally confirmed or studied.

The first target for an archaeal trans-encoded sRNA has been reported only very recently, for the highly abundant constitutively expressed $s \mathrm{RNA}_{162}$ in $M$. mazei, which is processed during growth [59]. Combining genetic approaches, genome-wide transcriptome analysis of $\mathrm{sRNA}_{162}$ mutants 
Figure 2 | mRNA targets of sRNA 162

(A) In silico predictions of the interaction with the mRNA MM2440-MM2441 target performed with IntaRNA [53], the RBS (ribosome-binding site) and the translational start codon are underlined. (B) Current working model; besides targeting mRNA MM2440-MM2441 (see A), the 5' region of sRNA 162 interacts with the 5' UTR of MM2442 mRNA.

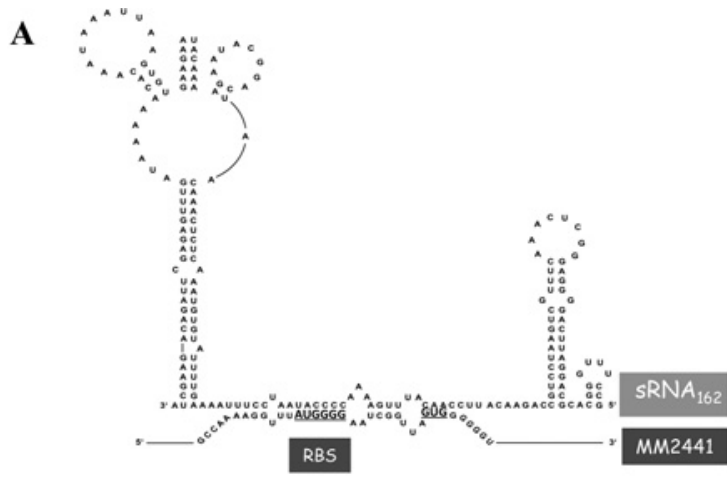

B
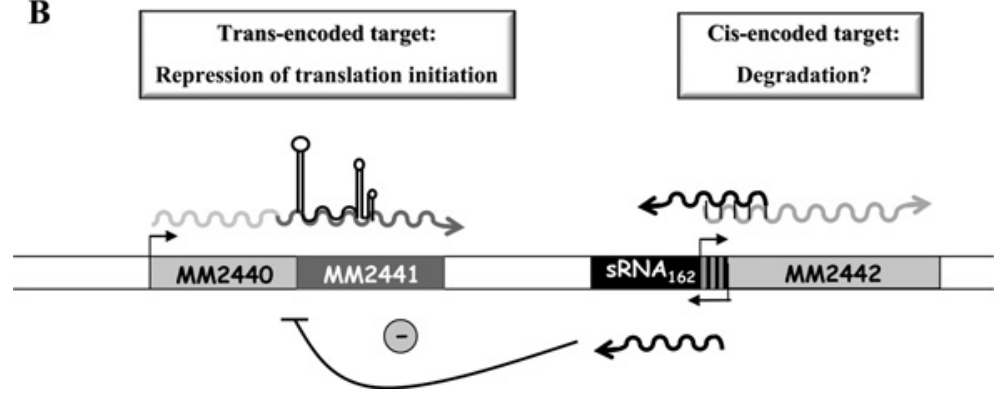

using microarrays and computational target predictions using the tool IntaRNA [53] identified a bicistronic mRNA (MM2440-MM2441) as trans-encoded target mRNA of $s \mathrm{RNA}_{162}$ [59]. The predicted interaction between $s \mathrm{RNA}_{162}$ and the target mRNA MM2440-2441 was verified further in vitro by mobility-shift assays and in vivo studying various mutant derivatives of $s \mathrm{RNA}_{162}$, demonstrating that the nonstructured single-stranded linker region of $s \mathrm{RNA}_{162}$ basepairs with the ribosome-binding site of MM2441 and with the translational start codon (Figure 2A). Masking the ribosomebinding site as well as the translational start probably leads to a dis-co-ordinated operon expression due to the sRNA-dependent translation inhibition of the second cistron (MM2441) coding for a transcriptional regulator (Figure 2). Further studies strongly indicate that $\mathrm{RNNA}_{162}$ is involved in the regulation of soluble methyltransferases expression patterns following the carbon source shift from methanol to trimethylamine probably due to translation inhibition of MM2441 [59]. Targeting the 3' UTR of mRNAs has been considered previously to be very likely for archaeal sRNAs, since several cellular processes in archaea are more similar to their eukaryotic than to their bacterial counterparts [60], thus the finding that $M$. mazei $\mathrm{sNA}_{162}$ is targeting the 5' UTR of its target (MM2441) was surprising and indicates that $s \mathrm{RNA}_{162}$ acts in a similar way to bacterial sRNAs. Moreover, in addition to the trans-encoded target
mRNA (MM2440-MM2441), evidence was obtained that the $5^{\prime}$ region of sRNA 162 also interacts with a second, cis-encoded, target, the 5' UTR of MM2442 mRNA [59]. This finding strongly indicates that $s \mathrm{RNA}_{162}$ acts as an antisense RNA on cis-encoded as well as on trans-encoded mRNAs (Figure 2B) most likely via two distinct domains, a mechanism that has not been shown for any sRNA in prokaryotes studied so far. MM2442 encodes a conserved protein of as yet unknown function in $M$. mazei, thus the physiological consequence of the potential down-regulation of MM2442 by sRNA 162 cannot easily be elucidated.

First experimental data for $H$. volcanii argue that sRNAs potentially target $3^{\prime}$ UTRs (J. Babski and J. Soppa, personal communication). If this is indeed the case, those recent studies on the biological function of archaeal sRNAs strongly suggest that archaeal sRNAs appear to target both the $5^{\prime}$ and the $3^{\prime}$ UTRs of their respective target mRNAs. Additional implication for an archaeal sRNA targeting a $3^{\prime}$ UTR has been obtained from a recent comparative RNA sequencing approach studying four Pyrobaculum strains [33]. Here a 65nt-long antisense transcript, asR3, has been identified that overlaps with the $3^{\prime}$ region of the tpi gene. Furthermore, a conserved structural element located close to the stop codon has been recognized in the $3^{\prime}$ region of the tpi gene (designated the 'tpi element'), the function of which might be early transcription termination or translation termination. 
Figure 3 | Cartoon of the potential targets of archaeal sRNAs

Targeting the 5' UTR has been demonstrated in M. mazei, and 3' UTR targeting has been suggested for Haloarchaea and Pyrobaculum strains. Targeting the CDS (coding sequence) or the presence of functional riboswitches are currently not experimentally verified in archaea. TT, transcription termination; RBS, ribosome-binding site.

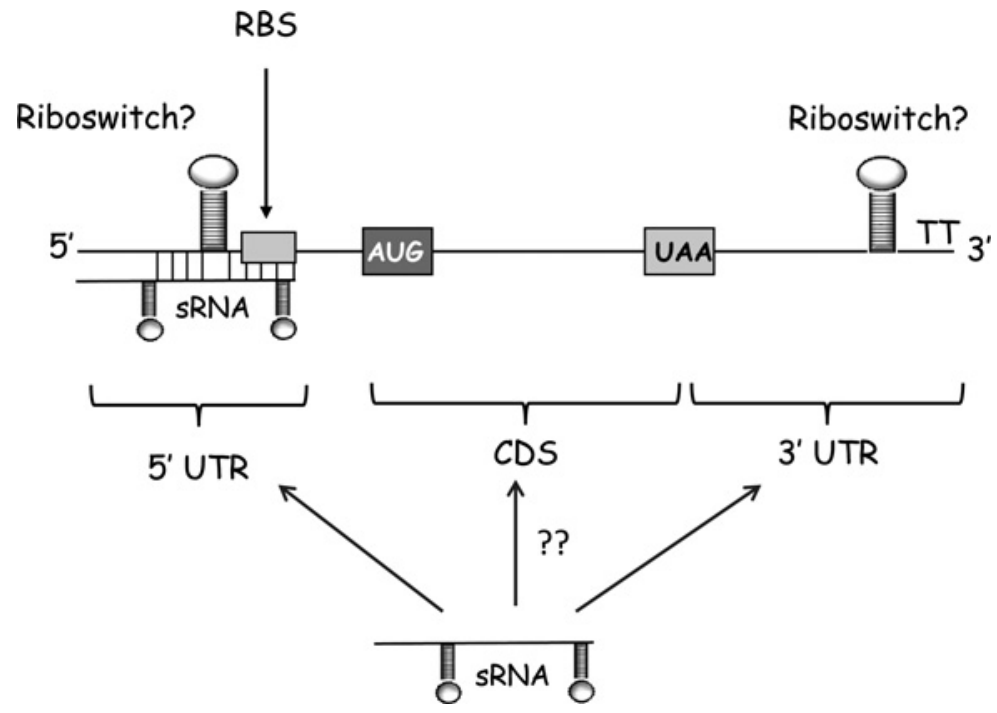

The formation of double-stranded RNA due to interaction of the tpi element with its associated antisense asR 3 might be able to compete with intramolecular structures of the mRNA and consequently might modulate the function of the highly conserved tpi element.

\section{Conclusion}

Despite the large number of archaeal sRNAs identified in recent years, studying biological functions and identifying targets have been started only recently mainly based on the lack of effective genetic tools. First evidence for an archaeal sRNA targeting the $5^{\prime}$ UTR of its targets has been recently obtained for $M$. mazei. Interacting with the ribosome-binding site and/or the translational start site indicates that this archaeal sRNA acts like its bacterial counterparts. Surprisingly, the respective sRNA targets the $5^{\prime}$ UTR of a cis-encoded and in addition a trans-encoded mRNA, strongly suggesting that cis-encoded asRNAs can have larger target regulons than previously estimated. Preliminary data obtained for Haloarchaea and Pyrobaculum strains strongly suggest that, besides 5' UTRs, 3' UTRs can also be targeted by archaeal sRNAs. Thus elucidation of the molecular regulatory mechanisms of archaeal ncRNAs including potential riboswitches emerges as a fascinating field in the future (Figure 3).

\section{Acknowledgements}

We thank the members of our laboratories for useful discussions, as well as Claudia Kiessling and jutta Kock for technical assistance.

\section{Funding}

This work was supported by the German Research Council (DFG) priority programme (SPP) 1258 'Sensory and Regulatory sRNAs in Prokaryotes' [grant numbers Schm1052/9-1 and Schm1052/9-2].

\section{References}

1 Gottesman, S. (2004) The small RNA regulators of Escherichio coli: roles and mechanisms. Annu. Rev. Microbiol. 58, 303-328

2 Romby, P., Vandenesch, F. and Wagner, E.G. (2006) The role of RNAs in the regulation of virulence-gene expression. Curr. Opin. Microbiol. $\mathbf{9}$, $229-236$

3 Waters, L.S. and Storz, G. (2009) Regulatory RNAs in bacteria. Cell 136 615-628

4 Guleria, P., Mahajan, M., Bhardwaj, J. and Yadav, S.K. (2011) Plant small RNAs: biogenesis, mode of action and their roles in abiotic stresses. Genomics, Proteomics Bioinformatics 9, 183-199

$5 \mathrm{Krol}$, J., Loedige, I. and Filipowicz, W. (2010) The widespread regulation of microRNA biogenesis, function and decay. Nat. Rev. 11, 597-610

6 Brantl, S. (2012) Acting antisense: plasmid- and chromosome-encoded sRNAs from Gram-positive bacteria. Future Microbiol. 7, 853-871

7 Mallory, A.C. and Vaucheret, H. (2006) Functions of microRNAs and related small RNAs in plants. Nat. Genet. 38 (Suppl.), S31-S36

8 Carthew, R.W. and Sontheimer, E.J. (2009) Origins and mechanisms of miRNAs and siRNAs. Cell 136, 642-655

9 Repoila, F. and Darfeuille, F. (2009) Small regulatory non-coding RNAs in bacteria: physiology and mechanistic aspects. Biol. Cell 101, 117-131

10 Brantl, S. (2009) Bacterial chromosome-encoded small regulatory RNAs. Future Microbiol. 4, 85-103

11 Storz, G., Vogel, J. and Wassarman, Karen M. (2011) Regulation by small RNAs in bacteria: expanding frontiers. Mol. Cell 43, 880-891

12 Brennan, R.G. and Link, T.M. (2007) Hfq structure, function and ligand binding. Curr. Opin. Microbiol. 10, 125-133

13 Dennis, P.P., Omer, A. and Lowe, T. (2001) A guided tour: small RNA function in archaea. Mol. Microbiol. 40, 509-519

14 Schattner, P. (2002) Searching for RNA genes using base-composition statistics. Nucleic Acids Res. 30, 2076-2082

15 Rozhdestvensky, T.S., Tang, T.H., Tchirkova, I.V., Brosius, J., Bachellerie, J.P. and Hüttenhofer, A. (2003) Binding of L7Ae protein to the K-turn of archaeal snoRNAs: a shared RNA binding motif for C/D and H/ACA box snoRNAs in archaea. Nucleic Acids Res. 31, 869-877 
16 Omer, A.D., Ziesche, S., Decatur, W.A., Fournier, M.J. and Dennis, P.P. (2003) RNA-modifying machines in archaea. Mol. Microbiol. 48, 617-629

17 Dennis, P.P. and Omer, A. (2005) Small non-coding RNAs in archaea. Curr. Opin. Microbiol. 8, 685-694

18 Klein, R.J., Misulovin, Z. and Eddy, S.R. (2002) Noncoding RNA genes identified in AT-rich hyperthermophiles. Proc. Natl. Acad. Sci. U.S.A. 99 $7542-7547$

19 Tang, T.H., Rozhdestvensky, T.S., d'Orval, B.C., Bortolin, M.L., Huber, H., Charpentier, B., Branlant, C., Bachellerie, J.P., Brosius, J. and Huttenhofer, A. (2002) RNomics in archaea reveals a further link between splicing of archaeal introns and rRNA processing. Nucleic Acids Res. 30, 921-930

20 Tang, T.H., Polacek, N., Zywicki, M., Huber, H., Brugger, K., Garrett, R., Bachellerie, J.P. and Hüttenhofer, A. (2005) Identification of novel non-coding RNAs as potential antisense regulators in the archaeon Sulfolobus solfatoricus. Mol. Microbiol. 55 , 469-481

21 Zago, M.A., Dennis, P.P. and Omer, A.D. (2005) The expanding world of small RNAs in the hyperthermophilic archaeon Sulfolobus solfatoricus. Mol. Microbiol. 55, 1812-1828

22 Phok, K., Moisan, A., Rinaldi, D., Brucato, N., Carpousis, A.J., Gaspin, C. and Clouet-d'Orval, B. (2011) Identification of CRISPR and riboswitch related RNAs among novel noncoding RNAs of the euryarchaeon Pyrococcus abyssi. BMC Genomics 12, 312

23 Jochl, C., Rederstorff, M., Hertel, J., Stadler, P.F., Hofacker, I.L., Schrettl, M., Haas, H. and Hüttenhofer, A. (2008) Small ncRNA transcriptome analysis from Aspergillus fumigatus suggests a novel mechanism for regulation of protein synthesis. Nucleic Acids Res. 36, 2677-2689

24 Sittka, A., Lucchini, S., Papenfort, K., Sharma, C.M., Rolle, K., Binnewies, T.T., Hinton, J.C. and Vogel, J. (2008) Deep sequencing analysis of smal noncoding RNA and mRNA targets of the global post-transcriptional regulator, Hfq. PLoS Genet. 4, e1000163

25 Yoder-Himes, D.R., Chain, P.S., Zhu, Y., Wurtzel, 0., Rubin, E.M., Tiedje, J.M. and Sorek, R. (2009) Mapping the Burkholderio cenocepocio niche response via high-throughput sequencing. Proc. Natl. Acad. Sci. U.S.A. $106,3976-3981$

26 Sharma, C.M. and Vogel, J. (2009) Experimental approaches for the discovery and characterization of regulatory small RNA. Curr. Opin. Microbiol. 12, 536-546

27 Jager, D., Sharma, C.M., Thomsen, J., Ehlers, C., Vogel, J. and Schmitz, R.A (2009) Deep sequencing analysis of the Methonosarcino mozei Go1 transcriptome in response to nitrogen availability. Proc. Natl. Acad. Sci. U.S.A. 106, 21878-21882

28 Wurtzel, O., Sapra, R., Chen, F., Zhu, Y., Simmons, B.A. and Sorek, R. (2010) A single-base resolution map of an archaeal transcriptome. Genome Res. 20, 133-141

29 Xu, N., Li, Y., Zhao, Y.T., Guo, L., Fang, Y.Y., Zhao, J.H., Wang, X.J., Huang, L. and GUo, H.S. (2012) Identification and characterization of small RNAs in the hyperthermophilic archaeon Sulfolobus solfataricus. PloS ONE $\mathbf{7}$ e35306

30 Straub, J., Brenneis, M., Jellen-Ritter, A., Heyer, R., Soppa, J. and Marchfelder, A. (2009) Small RNAs in haloarchaea: identification, differential expression and biological function. RNA Biol. 6, 281-292

31 Fischer, S., Benz, J., Spath, B., Jellen-Ritter, A., Heyer, R., Dorr, M., Maier, L.K., Menzel-Hobeck, C., Lehr, M., Jantzer, K. et al. (2011) Regulatory RNAs in Haloferax volcanii. Biochem. Soc. Trans. 39, 159-162

32 Heyer, R., Dorr, M., Jellen-Ritter, A., Spath, B., Babski, J., Jaschinski, K., Soppa, J. and Marchfelder, A. (2012) High throughput sequencing reveals a plethora of small RNAs including tRNA derived fragments in Holoferox volconii. RNA Biol. 9, 1011-1018

33 Bernick, D.L., Dennis, P.P., Lui, L.M. and Lowe, T.M. (2012) Diversity of antisense and other non-coding RNAs in archaea revealed by comparative small RNA sequencing in four Pyroboculum species. Front. Microbiol. 3, 231

34 Randau, L. (2012) RNA processing in the minimal organism Nonoarchoeum equitons. Genome Biol. 13, R63

35 Marchfelder, A., Fischer, S., Brendel, J., Stoll, B., Maier, L.K., Jager, D., Prasse, D., Plagens, A., Schmitz, R.A. and Randau, L. (2012) Small RNAs for defence and regulation in archaea. Extremophiles 16, 685-696

36 Lim, J., Thomas, T. and Cavicchioli, R. (2000) Low temperature regulated DEAD-box RNA helicase from the Antarctic archaeon, Methonococcoides burtonii. J. Mol. Biol. 297, 553-567

37 Opulencia, R.B., Bose, A. and Metcalf, W.W. (2009) Physiology and posttranscriptional regulation of methanol:coenzyme $M$ methyltransferase isozymes in Methanosarcino acetivorans C2A. J. Bacteriol. 191, 6928-6935
38 Anderson, K.L., Apolinario, E.E., MacAuley, S.R. and Sowers, K.R. (2009) A $5^{\prime}$ leader sequence regulates expression of methanosarcinal $\mathrm{CO}$ dehydrogenase/acetyl coenzyme A synthase. J. Bacteriol. 191 7123-7128

39 Lange, C., Zaigler, A., Hammelmann, M., Twellmeyer, J., Raddatz, G., Schuster, S.C., Oesterhelt, D. and Soppa, J. (2007) Genome-wide analysis of growth phase-dependent translational and transcriptional regulation in halophilic archaea. BMC Genomics 8, 415

40 Xia, Q., Hendrickson, E.L., Zhang, Y., Wang, T., Taub, F., Moore, B.C., Porat, I., Whitman, W.B., Hackett, M. and Leigh, J.A. (2006) Quantitative proteomics of the archaeon Methonococcus maripaludis validated by microarray analysis and real time PCR. Mol. Cell. Proteomics 5, 868-881

41 Brenneis, M., Hering, 0., Lange, C. and Soppa, J. (2007) Experimenta characterization of cis-acting elements important for translation and transcription in halophilic archaea. PLoS Genet. $\mathbf{3}$, e229

42 Weinberg, Z., Wang, J.X., Bogue, J., Yang, J., Corbino, K., Moy, R.H. and Breaker, R.R. (2010) Comparative genomics reveals 104 candidate structured RNAs from bacteria, archaea, and their metagenomes. Genome Biol. 11, R31

43 Brenneis, M. and Soppa, J. (2009) Regulation of translation in haloarchaea: $5^{\prime}$ - and $3^{\prime}$-UTRs are essential and have to functionally interact in vivo. PLoS ONE 4, e4484

44 Rother, M. and Metcalf, W.W. (2005) Genetic technologies for archaea. Curr. Opin. Microbiol. 8, 745-751

45 Ehlers, C., Jäger, D. and Schmitz, R.A. (2011) Establishing a markerless genetic exchange system for Methanosarcina mazei strain G01 for constructing chromosomal mutants of small RNA genes. Archaea 2011 439608

46 Said, N., Rieder, R., Hurwitz, R., Deckert, J., Urlaub, H. and Vogel, J. (2009) In vivo expression and purification of aptamer-tagged small RNA regulators. Nucleic Acids Res. 37 , e133

47 Corcoran, C.P., Rieder, R., Podkaminski, D., Hofmann, B. and Vogel, J. (2012) Use of aptamer tagging to identify in vivo protein binding partners of small regulatory RNAs. Methods Mol. Biol. 905, 177-200

48 Urban, J.H. and Vogel, J. (2009) A green fluorescent protein (GFP)-based plasmid system to study post-transcriptional control of gene expression in vivo. Methods Mol. Biol. 540, 301-319

49 Corcoran, C.P., Podkaminski, D., Papenfort, K., Urban, J.H., Hinton, J.C. and Vogel, J. (2012) Superfolder GFP reporters validate diverse new mRNA targets of the classic porin regulator, MicF RNA. Mol. Microbiol. 84 428-445

50 Mückstein, U., Tafer, H., Hackermuller, J., Bernhart, S.H., Stadler, P.F. and Hofacker, I.L. (2006) Thermodynamics of RNA-RNA binding. Bioinformatics 22, 1177-1182

51 Pichon, C. and Felden, B. (2008) Small RNA gene identification and mRNA target predictions in bacteria. Bioinformatics 24, 2807-2813

52 Tafer, H. and Hofacker, I.L. (2008) RNAplex: a fast tool for RNA-RNA interaction search. Bioinformatics 24, 2657-2663

53 Busch, A., Richter, A.S. and Backofen, R. (2008) IntaRNA: efficient prediction of bacterial sRNA targets incorporating target site accessibility and seed regions. Bioinformatics 24, 2849-2856

54 Ying, X., Cao, Y., Wu, J., Liu, Q., Cha, L. and Li, W. (2011) sTarPicker: a method for efficient prediction of bacterial sRNA targets based on a two-step model for hybridization. PLOS ONE 6 , e22705

55 Chitsaz, H., Salari, R., Sahinalp, S.C. and Backofen, R. (2009) A partition function algorithm for interacting nucleic acid strands. Bioinformatics 25 , i365-i373

56 Huang, F.W., Qin, J., Reidys, C.M. and Stadler, P.F. (2009) Partition function and base pairing probabilities for RNA-RNA interaction prediction. Bioinformatics 25, 2646-2654

57 Richter, A.S. and Backofen, R. (2012) Accessibility and conservation: general features of bacterial small RNA-mRNA interactions? RNA Biol. 9 954-965

58 Seemann, S.E., Richter, A.S., Gesell, T., Backofen, R. and Gorodkin, J. (2011) PETcofold: predicting conserved interactions and structures of two multiple alignments of RNA sequences. Bioinformatics 27, 211-219

59 Jäger, D., Pernitzsch, S.R., Richter, A.S., Backofen, R., Sharma, C.M. and Schmitz, R.A. (2012) An archaeal sRNA targeting cis- and trans-encoded mRNAs via two distinct domains. Nucleic Acids Res. 40, 10964-10979

60 Garrett, R.A. and Klenk, H.-P. (2007) Archaea: Evolution, Physiology, and Molecular Biology, Blackwell Publishing, oxford

Received 1 November 2012

doi:10.1042/BST20120280 